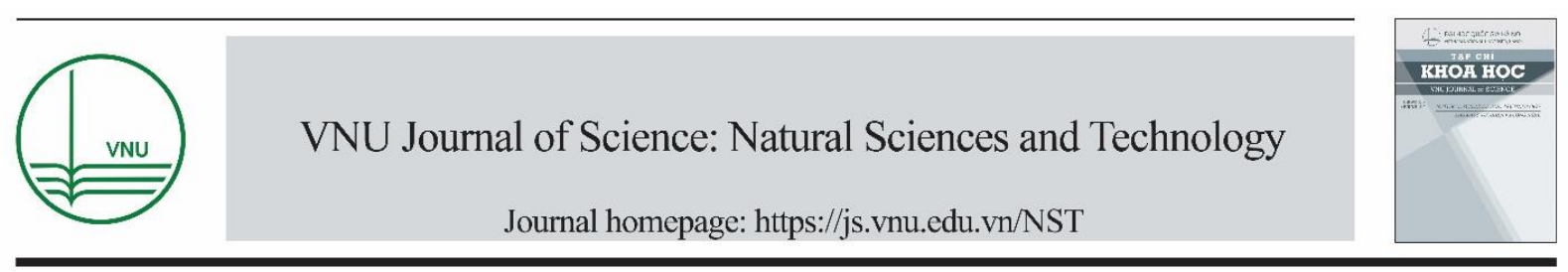

Original Article

\title{
Determination of the Earliest Time for Heat-shock Inducing Osteoporosis-like Phenotype in the rankl:HSE:CFP Medaka Fish Model for Osteoporosis
}

\author{
To Thanh Thuy ${ }^{*}$, Phan Cong Son, Tran Duc Long \\ VNU University of Science, 334 Nguyen Trai, Thanh Xuan, Hanoi, Vietnam
}

Received 10 August 2021

Revised 23 August 2021; Accepted 30 August 2021

\begin{abstract}
The transgenic medaka rankl:HSE:CFP expressing Rankl, a stimulator for osteoclastogenesis - the formation and activation of osteoclasts, bone "eating" cells, under the control of a heat inducible promotor, has been established as a model for osteoporosis to evaluate antiosteoporosis effects of substances. Transgenic larvae are usually heat-shocked for 90 minutes at $39{ }^{\circ} \mathrm{C}$ when they are at 9 days post fertilization (dpf) and osteoporosis-like phenotype is analysed when larvae are at $11 \mathrm{dpf}$. In this study, we investigated whether osteoporosis-like phenotype could be induced in the transgenic larvae when heat-shock was applied at earlier time points, namely when larvae were at $1,2,3,4,5$, or $6 \mathrm{dpf}$. The obtained results show that heat-shocks from the time point of $3 \mathrm{dpf}$ onwards resulted in osteoporosis-like phenotype, while heat-shock at 1 or $2 \mathrm{dpf}$ did not affect mineralized bone matrix in $11 \mathrm{dpf}$ larvae. These provide important evidence for study of onset of Rankl induced osteoclasts in fish and help improve experimental procedures using this fish model for osteoporosis.
\end{abstract}

Keywords: Medaka, heat-shock, osteoclasts, rankl, osteoporosis, fish model.

\section{Introduction}

Osteoporosis is a common bone disorder characterized by decrease in bone density and structure, resulting in elevated risk of bone fracture. This disease is most prevalent in middle aged and old people. It is also resulted from some medications and unhealthy lifestyle $[1,2]$. Globally, there are more than 200 million

\footnotetext{
* Corresponding author.

E-mail address: tothanhthuy@hus.edu.vn

https://doi.org/10.25073/2588-1140/vnunst.5296
}

people diagnosed with the disease and the number of affected people is increasing dramatically due to aging population and lack of effective and safe drugs and therapies [2].

Animal models are always important for research to develop better drugs for osteoporosis. Besides commonly used mammals including mouse, rats, rabbits, and primates; recently some fish species, especially the medaka (Oryzias latipes) have also been used for this purpose and shown great potential [3-6].

Medaka owns biological characteristics highly suitable for use as an experimental 
animal model for numerous human diseases $[4,7,8]$. For bone studies, mechanisms underlying bone biology and pathology in medaka are highly comparable to those in humans at the molecular and cellular levels [6, 9-11]. Medaka is externally fertilized and has a small genome, facilitating genetic manipulation for generation of transgenics and mutants for studies on gene functions [7, 12]. Their embryos are transparent allowing observing and imaging bone and live bone cells in real time $[6,13,14]$. Furthermore, there are benefits of using fish over mammals such as simple maintenance with low cost, fast growing and short generation time, large number of offsprings, feasible requirements of bioethics [7].

In 2012, To et al., generated a rankl:HSE:CFP transgenic medaka fish for use as a model of osteoporosis. This transgenic fish expresses Rankl (Receptor activator of nuclear factor kappa-B ligand), a key factor promoting formation, differentiation, and activation of osteoclasts, bone resorbing/eating cells. Expression of exogenous Rankl is controlled by the heat-inducible promoter; thus, when larvae are subjected to a heat shock, Rankl is expressed and osteoclasts are formed, resulting in damage in mineralized bone matrix of larvae, an osteoporosis-like phenotype. Bone damage is most evident in the vertebrae column of larvae [6]. This transgenic fish was then brought to our laboratory at VNU University of Science, maintained and segregated into sublines that display bone damage at neural arches at different extents for use to screen for anti-osteoporosis substances [15, 16]. For this use, transgenic larvae were heat-shocked for 90 minutes at $39{ }^{\circ} \mathrm{C}$ when they were at 9 days post fertilization (dpf) and their osteoporosis-like phenotypes were analyzed when they were at 11 dpf. In this study, we investigated whether osteoporosis-like phenotype is induced in $11 \mathrm{dpf}$ larvae when larvae are heat-shocked at earlier time points. Osteoporosis like phenotype is determined through Index of bone mineralization $\left(\mathrm{I}_{\mathrm{M}}\right)$. $\mathrm{I}_{\mathrm{M}}$ value is the total length of mineralized neural arches of the first 15 vertebrae of the larva. The level of bone damage of a larva is therefore inversely corelated with the $\mathrm{I}_{\mathrm{M}}$, meaning the higher the $\mathrm{I}_{\mathrm{M}}$ value, the lower the level of bone damage of larvae [5]. Data from this study will provide important insight into the onset of Rankl-induced osteoclast formation and help improve application of this transgenic fish.

\section{Methodology}

\subsection{Fish Lines and Fish Maintenance}

For this study, wild type fish and c6 rankl:HSE:CFP subline transgenic fish whose osteoporosis-like phenotype was only observed in neural arches were used [15]. The c6 rankl:HSE:CFP transgenic fish/embryos are hereafter referred to as Rankl fish/embryos/larvae. Hemizygous Rankl embryos were obtained by crossing homozygous Rankl fish with wild type fish.

Fish were raised and maintained at temperature of $28-30{ }^{\circ} \mathrm{C}$ with light cycles of 14 hour light and 10 hour dark [5, 16]. As a heat-shock inducible promotor in this transgenic fish controls expression of both Rankl and cyan fluorescent reporter protein $\mathrm{CPF}$, transgenic embryos were screened for by CFP signal at 11 days post fertilization (dpf), before bone staining.

\subsection{Staining of Mineralized Bone Structures}

Fish larvae at $11 \mathrm{dpf}$ were fixed and stained with alizarin red (Sigma A5533) to visualize mineralized matrix as previously described $[5,6]$.

\subsection{Imaging}

For $11 \mathrm{dpf}$ alizarin red bone-stained larvae, images were acquired using an Axiovert $100 \mathrm{M}$ microscope (Carl Zeiss, Germany). Larvae were laid laterally on a slide between two stacks of 3 -coverslip and imaged multiple times to cover at least the first 15 vertebrae. Those images 
were then stitched together to form a complete image of a larva.

\subsection{Quantification of Level of Bone Mineralization and Bone Mineralization Damage}

Using the $\mathrm{I}_{\mathrm{M}}$ method published previously, level of bone mineralization and bone mineralization damage of the larvae were determined via Index of bone mineralization $\left(\mathrm{I}_{\mathrm{M}}\right)$ and Index of mineralization damage $\left(\mathrm{I}_{\mathrm{D}}\right)$, respectively [5]. Because bone damage occurs mainly in the mineralized neural arches of the Rankl larvae so these bone structures were chosen as representative bone structures to be analyzed in larvae. Lengths of their first 15 mineralized neural arches were measured using ImageJ software (NIH).

$I_{M}$ is defined as the sum of lengths of the first 15 mineralized neural arches and calculated by the formular: $\mathrm{I}_{\mathrm{M}}=\sum_{k=1}^{15} L$, where $\mathrm{k}$ is the ordinal number of neural arch and $\mathrm{L}$ is the length of each arch. Based on the $\mathrm{I}_{\mathrm{M}}$ of Rankl larvae and wild type larvae, Index of mineralization damage $\mathrm{I}_{\mathrm{D}}$ of Rank larvae was calculated by the formular: $\mathrm{I}_{\mathrm{D}}=\left[\mathrm{I}_{\mathrm{M} \text { (WT) }}-\mathrm{I}_{\mathrm{M} \text { (Rank) })}\right] / \mathrm{I}_{\mathrm{M} \text { (WT) }} \times 100 \%$, where $\mathrm{I}_{\mathrm{D}}$ is the percentage of mineralization damage of neural arches of a larva, $\mathrm{I}_{\mathrm{M}}$ (wT) is the Index of bone mineralization of wild-type larvae, and $\mathrm{I}_{\mathrm{M} \text { (Rankl) }}$ is Index of bone mineralization of the corresponding Rankl larvae. $I_{M}$ is inversely correlated to $I_{D}$.

\subsection{Statistical Analysis}

Student t-tests (two-tailed, unequal variance) or one-way ANOVA followed by Tukey's multiple comparison test were used to compare different experimental groups and to determine significance with Prism 5 software (GraphPad Software Inc., San Diego, CA). Differences were considered statistically significant when $\mathrm{p}<0.05$ (marked with one asterisk $*)$; or $\mathrm{p}<0.001(* * *)$, or $\mathrm{p}<0.0001$ $(* * * *)$. Results are presented as mean \pm S.E.M.

\subsection{Ethical Aspects}

All fish experiments in this study were performed in accordance with the animal welfare laws and guidelines from Dinh Tien Hoang Institute of Medicine, Hanoi, Vietnam (Approval number: IRB-AR.002).

\section{Results and Discussion}

Hemizygous embryos and larvae offspring of c6 homogenous Rankl fish and a wild type which show bone damage only in neural arches were used for this study. We firstly examined the osteoporosis-like phenotype of c6 Rankl fish as previously described [15] and then heat-shocked the fish at earlier time points of 1 , $2,3,4,5$, or $6 \mathrm{dpf}$ and analyzed their mineralized bone matrix to check for their osteoporosis phenotype when fish were at $11 \mathrm{dpf}$.

\subsection{Osteoporosis-like Phenotype of $11 \mathrm{dpf}$ Rankl Larvae Induced by Heat-shock at 9 dpf}

A group of 9 dpf Rankl larvae $(n=40)$ were subjected to heat-shock at $39{ }^{\circ} \mathrm{C}$ for 90 minutes and raised until they were $11 \mathrm{dpf}$ to be fixed for bone staining with alizarin red. For control, a group of wild type larva $(n=33)$ with similar experimental procedures was included. Results are shown in Figure 1.

As observed in Figure 1, the $11 \mathrm{dpf}$ wild type larva shows intact mineralized bone structures of the head, the vertebral column, and the tail (Figures 1A2, A4). Each vertebra consists of a vertebral body (asterisk in Figure 1A4) and a pair of neural arches, of which one is observed from lateral side (arrow in Figure 1A4). Neural arches of wild type larva are intact while these bone structures in the Rankl larva are mostly destructed. Levels of mineralization of the two larva groups were then determined by IM as described in 2.3. Resulted mean IM values of wild type and Rankl larva groups were calculated as 3147 and 1643, respectively. From these data, the Index of bone mineralization damage ID of the Rankl larvae (see formula for ID index in 2.3) was calculated as $47.8 \%$. This means that these $11 \mathrm{dpf}$ Rankl larvae lost $47.7 \%$ of their mineralized neural arches, a level of bone loss equivalent to what previously observed in this fish line. 

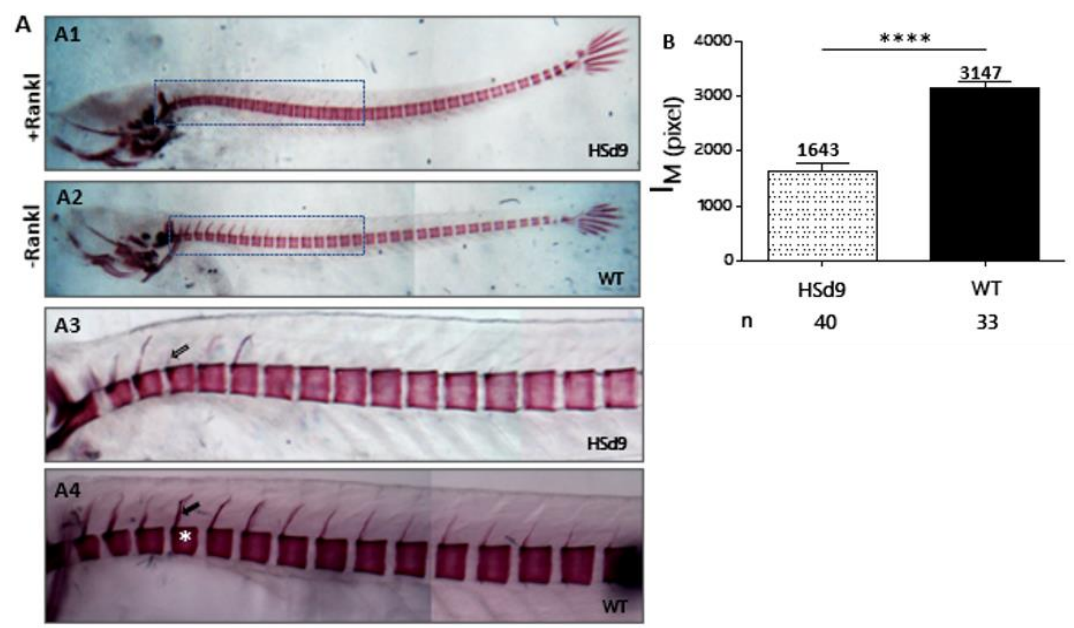

Figure 1. Osteoporosis-like phenotype of Rankl larvae at $11 \mathrm{dpf}$ induced by a 90 -minute heat-shock at $39{ }^{\circ} \mathrm{C}$ when larvae were at $9 \mathrm{dpf}$. A. Images of alizarin red-stained of mineralized bone structures of a $11 \mathrm{dpf}$ Rankl (+Rankl, HSd9) (A1) and a $11 \mathrm{dpf}$ wild type (-Rank1, WT) (A2) larvae, both heat-shocked at 9 dpf. A3, A4: higher magnification of the first 15 vertebrae of the two larvae boxed in A1, A2, respectively. Black arrow (in A4) indicates intact neural arch, asterisk notes an intact vertebral body, white arrow (in A3) indicates a damaged neural arch. B. Mean mineralization index $\mathrm{I}_{\mathrm{M}}$ of wild-type (WT) and Rankl larvae. $\mathrm{n}$ : number of larvae in corresponding larva group $(* * * *) \mathrm{p}<0.0001$.

\subsection{Heat-shock at 1 or 2 dpf Did Not Induce Osteoporosis-like Phenotype in 11 dpf Rankl Larvae}

Figure 2 shows alizarin red stained of the first 15 vertebrae of $11 \mathrm{dpf}$ Rankl larvae when being heat-shocked at 1 (HSd1) or 2 (HSd2) dpf, and of $11 \mathrm{dpf}$ WT control larvae. We did not observe damage in mineralized bone structures of vertebrae of the Rankl larvae, neither in the vertebral bodies, nor in the neural arches (Figure 2A).

There is no significant difference in mean $\mathrm{I}_{\mathrm{M}}$ values of Rankl larva groups (2887.94 for larvae heat-shocked at $1 \mathrm{dpf}-\mathrm{HSd} 1$ and 2652.53 for larvae heat-shocked at 2 dpf-HSd2) and wild type larva group (2887.9-WT) (Figure 2B).
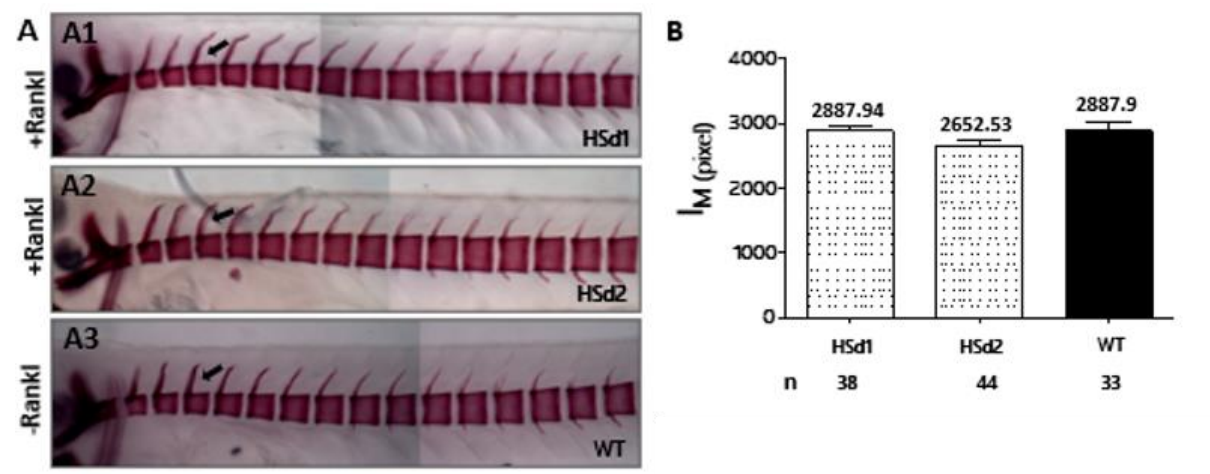

Figure 2. Heat-shock at 2 or $3 \mathrm{dpf}$ did not affect mineralized bone structures of $11 \mathrm{dpf}$ Rankl larvae. A. Representative images of the first 15 vertebrae of $11 \mathrm{dpf}$ Rankl larvae which were heat-shocked at $1 \mathrm{dpf}(\mathrm{A} 1, \mathrm{HSd} 1)$ and $2 \mathrm{dpf}(\mathrm{A} 2, \mathrm{HSd} 2)$ and of a wild type larva (A3, WT). B. Mean mineralization index $\mathrm{I}_{\mathrm{M}}$ of the three larva groups. Black arrows indicate intact neural arches of larvae in the three groups. 
Thus, heat-shocks applied at 1 or $2 \mathrm{dpf}$ could not induce osteoporosis phenotype when Rankl larvae reached 11 days of age. Heat-shock at these time points ensures expression of exogenous Rankl in the larvae [6]. Rankl is an important stimulator for formation and activity of osteoclasts from osteoclast precursors. That early exogenous Rankl expression did not affect mineralized bone structures in $11 \mathrm{dpf}$ larvae indicates that osteoclast precursors have not yet appeared at such early time. Moreover, exogenous Rankl protein may be degraded and cannot trigger osteoclastogenesis when osteoclast precursors appear later. Possible explanation may relate to the onset time for formation of head kidney of the fish. As in mammals, osteoclast progenitors in bony fish also derived from hematopoietic stem cells. However, in bony fish, hematopoietic stem cells are not present in the bone marrow but located in the head kidney [17]. In medaka, the head kidney appears at one day of age and its

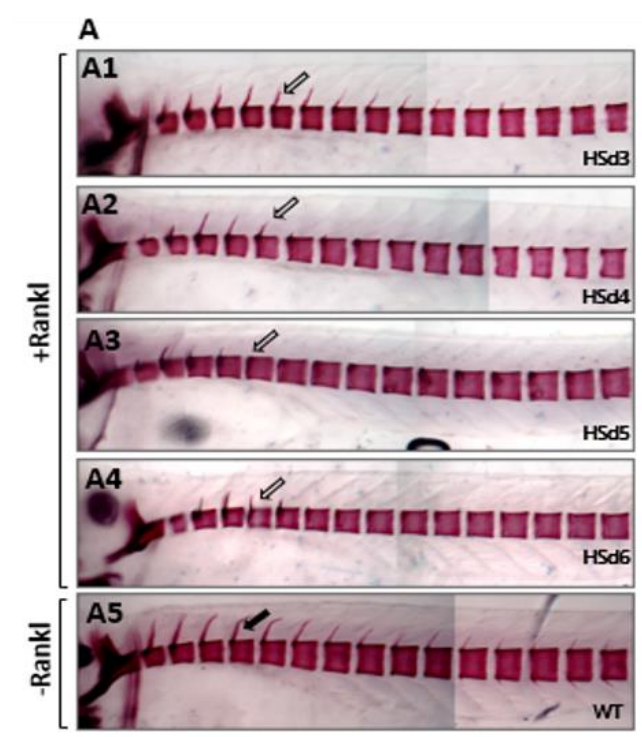

development is completed when the fish is three days old [7]. It is possible that during the early development of this structure, osteoclast progenitors are not yet formed, so despite the presence of Rankl, osteoclasts are not formed and no bone resorptive activity occurs and mineralized bone structures of the $11 \mathrm{dpf}$ larvae remained intact as observed.

\subsection{Heat-shock from 3 dpf Onwards Can Cause Osteoporosis-like Phenotype in Rankl Larvae}

We observed bone damage of 11 dpf Rankl larvae when they were heat-shocked at 3, 4, 5, or 6 dpf (Figure 3). Neural arches of the Rankl larvae were destructed to different extents when the larvae were heat-shocked at these different time points (white arrows in Figures 3 A1-A4). Mean $I_{M}$ values of larva groups show that level of bone mineralization of neural arches decreased when larvae were heat-shocked at later time points (Figure 3B).

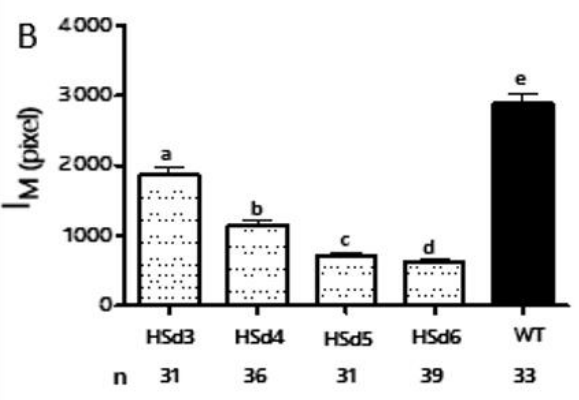

Figure 3. Heat-shocks applied to Rankl larvae when they were 3 days of age onwards could induce osteoporosis-like phenotype. A. Representative images of the first 15 vertebrae of $11 \mathrm{dpf}$ Rankl larva groups which were heat-shocked (+Rankl) at $3 \mathrm{dpf}(\mathrm{A} 1, \mathrm{HSd} 3), 4 \mathrm{dpf}(\mathrm{A} 2, \mathrm{HSd} 4), 5 \mathrm{dpf}(\mathrm{A} 3, \mathrm{HSd} 5)$, or $6 \mathrm{dpf}(\mathrm{A} 4, \mathrm{HSd} 6)$ and of a wild type group (A5, WT). Black arrows indicate intact neural arches of wild type larvae, white arrows point to damaged neural arches to different extents of Rankl larvae. B. Mean mineralization index $\mathrm{I}_{\mathrm{M}}$ of all larva groups. A-e: mean $\mathrm{I}_{\mathrm{M}}$ values of corresponding larva group $(1860.16,1151.79,701.328,620.716,2887.9)$. $\mathrm{a} \ddagger \mathrm{b}, \mathrm{c}, \mathrm{d}, \mathrm{e}(\mathrm{p}<0.001), \mathrm{b}+\mathrm{c}(\mathrm{p}<0.01), \mathrm{b}+\mathrm{d}, \mathrm{e}(\mathrm{p}<0.001)$, e $\ddagger \mathrm{c}, \mathrm{d}(\mathrm{p}<0.001)$ ( $\$$ indicates statistical differences between presented values that were identified by one-way ANOVA with Turkey post hoc test). $\mathrm{n}$ : number of larvae in corresponding group. 
As mentioned above, three days of age is the time when the formation of head kidney is complete [7]. Possibly from this time point onwards, hematopoietic progenitor cells that give rise to osteoclast precursors already present and differentiate into osteoclasts upon heat-shock induced Rankl expression. The number of osteoclast precursors increased over time as the larvae were 4,5 , or 6 days of age so that more osteoclasts were formed, resulting in lower $\mathrm{I}_{\mathrm{M}}$ value of the larvae. Thus, three days of age is the earliest time point when heat-shock could induce osteoporosis phenotype of the larvae. Interestingly, pattern of mineralized neural arche damage of the Rankl larvae heat-shocked at $3 \mathrm{dpf}$ is different from those of larvae heat-shocked at later time points (at 4, 5, or $6 \mathrm{dpf}$ ). Larvae heat-shocked at $3 \mathrm{dpf}$ showed gradually increasing damage of neural arches along anterior-posterior axis of the vertebral column (Figure 3A1) while in larvae heats-shocked at other time points, neural arches were damaged randomly, regardless of their position (Figure 3 A2-A4).

\subsection{Level of Bone Mineralization Damage in the Rankl Larvae}

From the mean $\mathrm{I}_{\mathrm{M}}$ values of all Rankl larva groups and of wild-type larva group of the study, Indexes of mineralization damage $I_{D}$ of Rankl larva groups (see the formular for $I_{D}$ in 2.3) heat-shocked at 1, 2, 3, 4, 5, or 6 days of age were calculated and compared to $\mathrm{I}_{\mathrm{D}}$ of the Rankl larvae heat-shocked at 9 days of age (Figure 4).

Figure 4 shows that earliest time point to heat-shock the larvae to induce bone damage and osteoporosis-like phenotype is when larvae are $3 \mathrm{dpf}$. Level of bone damage increases when larvae are heat-shocked at later time points (at 4, 5, or $6 \mathrm{dpf}$ ). This suggests that the number of osteoclast precursors was increasing over this period of time. Moreover, it seems that the level of bone damage is maximal when larvae are heat-shocked at 5 or $6 \mathrm{dpf}$ (that causes 77.17 or $80.06 \%$ loss of mineralized neural arches, respectively), as to heat-shock the larvae at 9 dpf could induce only $47.8 \%$ bone loss, the level nearly comparable to that of larvae heatshocked at $3 \mathrm{dpf}$ (32.87\%).

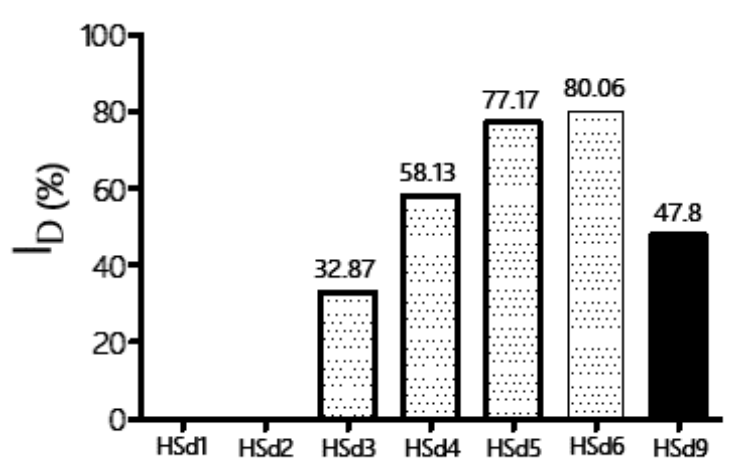

Figure 4. Indexes of mineralization damage $I_{D}$ of Rankl larva groups which were heat-shocked at 1,2 , $3,4,5,6$, or 9 days of age. HSd1, HSd2, HSd3, HSd4, HSd5, HSd6, HSd9: heat-shock time points when larvae reached one, two, three, four, five, six or nine days of age.

This may be due to onset and accumulation of Rankl-induced osteoclasts and the onset of mineralized neural arches in the larvae during the period of development of the examined larvae. As first mineralized neural arches were observed when the larvae were at 7-10 days of age [18], there was more time for Rankl-induced osteoclasts to resorb bone in larvae that were heat-shocked at 5 or $6 \mathrm{dpf}$ than larvae heat-shocked at $9 \mathrm{dpf}$.

These results show that osteoporosis-like phenotype can be induced by heat-shocking larvae as early as $3 \mathrm{dpf}$. Moreover, severity of the osteoporosis-like phenotype can be achieved by choosing suitable heat-shock time points. Heat-shocking larvae at 3 or $9 \mathrm{dpf}$ would result in moderate osteoporosis-like phenotype while heat-shocking larvae at 5 or $6 \mathrm{dpf}$ would result in most severe osteoporosis-like phenotype. Severity of osteoporosis-like phenotype would contribute to screening substances with different efficacies on osteoporosis.

\section{Conclusion}

Time points for heat-shock can influence level of bone damage in the $11 \mathrm{dpf}$ transgenic 
Rankl medaka model for osteoporosis. Heat-shock in earliest stages when larvae were at 1 or $2 \mathrm{dpf}$ did not affect larvae bone mineralization. Earliest time point for heat-shock that could induce bone damage and osteoporosis-like phenotype in the larvae was $3 \mathrm{dpf}$ (with $\mathrm{I}_{\mathrm{D}}$ of $32.87 \%$ ). Level of bone damage was highest when larvae were heat-shocked at five or six days of age ( $I_{D}$ of $77.17 \%$, or $80.06 \%$, respectively), while heat-shock at 9 days of age could induce $47.86 \%$ bone loss. These results are important evidence for onset of Rankl induced osteoclast formation in fish and may help improve experimental procedures using this transgenic fish.

\section{Acknowledgements}

We sincerely thank Assoc. Prof. Dr. Nguyen Lai Thanh and the Center for Life Science Research, Faculty of Biology for providing microscope equipment throughout the course of the study.

\section{References}

[1] T. Rachner, S. Khosla, L. Hofbauer, A. Manuscript, New Horizons in Osteoporosis, Lancet, Vol. 377, No. 9773, 2011, pp. 1276-1287.

[2] J. A. Cauley, Osteoporosis: Fracture Epidemiology Update 2016, Curr, Opin, Rheumatol, Vol. 29, No. 2 , 2017, pp. 150-156.

[3] T. Komori, Animal Models for Osteoporosis, Eur, J. Pharmacol, Vol. 759, 2015, pp. 287-294.

[4] P. E. Witten, M. P. Harris, A. Huysseune, C. Winkler, Mall Teleost Fish Provide New Insights into Human Skeletal Diseases, Methods Cell Biol, Vol. 38, 2017, pp. 321-346.

[5] C. V. Pham, T. T. Pham, T. T. Lai, D. C. Trinh, H. V. M. Nguyen, T. T. M. Ha et al., Icariin Reduces Bone Loss in a Rankl-induced Transgenic Medaka (Oryzias Latipes) Model for Osteoporosis, J. Fish Biol, Vol. 98, No. 4, 2021, pp. 1039-1048.

[6] T. T. To, P. E. Witten, J. Renn, D. Bhattacharya, A. Huysseune, C. Winkler et al., Rankl-induced Osteoclastogenesis Leads to Loss of Mineralization in a Medaka Osteoporosis Model, Vol. 139, No. 1, 2012, pp. 141-150.
[7] K. Naruse, M. Tanaka, H. Takeda, Medaka - A Model for Organogenesis, Human Disease, and Evolution, Science, 2011, pp. 132.

[8] C. Y. Lin, C. Y. Chiang, H. J. Tsai, Zebrafish and Medaka: New Model Organisms for Modern Biomedical Research, J. Biomed, Sci, Vol. 23, 2016, pp. 19.

[9] M. Chatani, Y. Takano, A. Kudo, Osteoclasts in Bone Modeling, as Revealed by in Vivo Imaging, are Essential for Organogenesis in Fish, Dev, Biol, Vol. 360, No. 1, 2011, pp. 96-109.

[10] A. Büttner, T. T. To, C. Winkler, S. J. H. Chan, J. Renn, A Col10a1_nlGFP Transgenic Line Displays Putative Osteoblast Precursors at the Medaka Notochordal Sheath Prior to Mineralization, Dev, Biol, Vol. 381, No. 1, 2013, pp. 134-143.

[11] T. T. To, P. E. Witten, A. Huysseune, C. Winkler, An Adult Osteopetrosis Model in Medaka Reveals the Importance of Osteoclast Function for Bone Remodeling in Teleost Fish, Comp, Biochem, Physiol, Part - C Toxicol, Pharmacol., Vol. 178, 2015, pp. 68-75.

[12] M. Kasahara, K. Naruse, S. Sasaki, Y. Nakatani, W. Qu, B. Ahsan et al., The Medaka Draft Genome and Insights into Vertebrate Genome Evolution, Nature, Vol. 447, 2007, pp. 714-719.

[13] T. Yu, P. E. Witten, A. Huysseune, A. Buettner, T. T. To, C. Winkler, Live imaging of Osteoclast Inhibition by Bisphosphonates in a Medaka Osteoporosis Model, Dis, Model, Mech, Vol. 9, No. 2, 2016, pp. 155-163.

[14] Q. T. Phan, W. H. Tan, R. Liu, S. Sundaram, A. Buettner, S. Kneitz et al., Cxc191 and Cxcr3.2 Regulate Recruitment of Osteoclast Progenitors to Bone Matrix in a Medaka Osteoporosis Model, Proc, Natl, Acad, Sci, USa, Vol. 117, No. 32, 2020, pp. 19276-19286.

[15] V. C. Pham, T. T. Pham, T. H. Nguyen, D. L. Tran, T. T. To, Segregation of rankl:HSE:CFP Medaka Transgenic Fish Line for Use as Osteoporosis Models, VNU Journal of Science: Natural Sciences and Technology, Vol. 31, No. 4S, 2015, pp. 24-34 (in Vietnamese).

[16] T. N. Lai, T. T. Pham, V. C. Pham, D. L. Tran, T. T. To, Stability of the Transgene Rankl in the rankl:HSE:CFP Transgenic Medaka Fish Used as a Model for Osteoporosis, Vietnam J. Physiol, Vol. 19, No. 2, 2015, pp. 10-17 (in Vietnamese).

[17] A. Apschner, S. Schulte-Merker, P. E. Witten, Not All Bones are Created Equal - using Zebrafish and Other Teleost Species in Osteogenesis Research, Methods Cell Biol, Vol. 105, No. 239, 2011, pp. 255.

[18] T. T. Pham, V. C. Pham, D. L. Tran, T. T. To, Bone Mineralization Development in Medaka (Oryzias Latipes), Vietnam J. Physiol, Vol. 19, No. 3, 2015, pp. 8-16 (in Vietnamese). 